\title{
PLOW Filter for Color Image Denoising
}

\author{
Jency Thomas \\ Department of Computer Science \& Engineering \\ Federal Institute of Science \& Technology \\ Angamaly, Ernakulam Dt., Kerala
}

\author{
Remya S \\ Department of Computer Science \& Engineering \\ Federal Institute of Science \& Technology \\ Angamaly, Ernakulam Dt., Kerala
}

\begin{abstract}
In this paper, a denoising approach, which exploits patchredundancy for removing Gaussian noise from RGB color images is described. Both geometrical and photometrical similarity of image patches have to be considered for learning the parameters of this Patch-based Locally Optimal Weiner(PLOW) filer. K-means clustering,with LARK(Locally Adaptive Regression Kernel) features, is used to identify the geometrically similar patches. As opposed to traditional color image denoising approaches, that perform denoising in each color channel independently, this method performs denoising in the luminance-chrominance color space and thus exploits correlation across color components. Since the luminance component, $\mathrm{Y}$, contains most valuable image features such as objects, shades, textures, edges and patterns etc., the information from the luminance component is only needed for clustering. Experimental results show that the denoising performance of the proposed method is better in terms of both peak signal-to-noise ratio and subjective visual quality.
\end{abstract}

\section{Keywords:}

Image denoising, Color Image restoration, Gaussian noise.

\section{INTRODUCTION}

In modern world digital images have a vital role in applications such as magnetic resonance imaging, satellite vision, computer tomography, astronomy etc. Visual information contained in an image may be corrupted by noise, which is an undesirable effect caused by random variation in brightness or color information in the image. Usually noise is introduced in to images during image acquisition and transmission. Due to faster shutter speeds and higher density of image sensors (pixels) higher levels of noise [1] is introduced into the captured image. It must then be processed by denoising algorithms to obtain an image of acceptable quality. The challenge of any image denoising algorithm is to reduce noise artifacts, while retaining details such as edges and texture in the image, even though the image is highly affected by noise.

The added noise will be of different types based on their pattern and probabilistic characteristics, such as Gaussian noise, impulse noise and speckle noise etc. Usually, noise in digital images is found to be additive in nature that contain equal power in the whole bandwidth and having an amplitude with Gaussian probability distribution. Such type of noise is referred to as Additive White Gaussian Noise (AWGN). Impulse noise can be either random valued noise[2] or salt and pepper noise[3]. In an image corrupted by salt- and-pepper noise, the noisy pixels will have either salt value (gray level-255) or pepper value (gray level-0)and it appears as black and white spots on the images. If the image is corrupted by random valued impulse noise, noisy pixel can take any gray level value from 0 to 255 and the noise is randomly distributed over the entire image. Here the probability of occurrence of any gray level value as noise will be same. The speckle noise [4] which is a multiplicative noise is usually observed in the ultrasound medical images and it degrades the quality of the Active Radar and Synthetic Aperture Radar (SAR) images.

Image denoising is a challenging task as the denoising algorithm depends upon the type of noise added to the image. The two basic approaches to image denoising are, namely spatial filtering and transform domain filtering. In spatial filtering each pixel value (u, $\mathrm{v}$ ) is changed by a function of the intensities of pixels in a neighborhood of $(u, v)$. In the case of transform domain filters, generally, the basis of signal space is changed to aid some processing on the image data; i.e., an image is first converted into transform domain and then the transform coefficients are altered to reduce noise. The commonly used methods of noise removal include NLM (Nonlocal means)filter [5] which takes the advantage of the high degree of redundancy of any natural image, Total Variation (TV) method, Shrinkage models and different transforms.

Among the above mentioned, Gaussian noise is the most common type of noise and it is very difficult to suppress AWGN (additive white Gaussian noise) as it corrupts almost all the pixels in the image. Linear spatial domain filters such as mean and wiener filters $[6,7]$ were the common means of suppressing AWGN, during the early days. Though linear techniques possess mathematical simplicity, they have the disadvantage of yielding blurring effect and they do not perform well in the presence of signal dependent noise. Wavelet based denoising has always considered as a successful and powerful tool for image denoising, since the use of wavelets successfully removes noise while preserving the signal characteristics, regardless of its frequency content. So many wavelet based denoising methods have been developed over the past years, among them wavelet thresholding is the most common method where the signal is decomposed into its approximation (low-frequency) and detail (high-frequency) sub bands; then the detail sub bands are processed with hard or soft thresholding operations, since the high frequency coefficients are mostly due to noise. Important issue here is to find the best signal representation and the proper threshold since threshold determines whether or not to keep the wavelet coefficient. 
Another popular denoising method developed in recent past is the bilateral filter [8] which is an edge-preserving and noise reducing smoothing filter. Here the intensity value at each pixel in an image is replaced by a weighted average of intensity values from neighboring pixels. Here weight is based on a Gaussian distribution and the weights depend not only on spatial distance (Euclidean distance) but also on the intensity distance (radiometric differences). Hence, edges are preserved in these types of filters while retaining its edges. Other approaches for image denoising are curvelet thresholding, PCA-based denoising and independent component analysis. PCA-based denoising found to be competitive with the state-of-theart denoising algorithms, particularly for large images and moderate signal-to-noise ratios. In the case of ICA transform [9] data is represented as the statistically independent random variables.

In real world, most images are stored either in color or in various layers of gray scale. The most denoising methods are developed for gray-scale images and only a few concentrates on denoising color images. Grayscale image denoising can be straightforwardly extended to color images by applying it to each color component independently. Some other methods perform denoising by explicitly modeling the color information at each pixel. Run time overhead of these methods are quite high. However, frequencies at which the human eye perceives each of the red, green, and blue (RGB) colors have considerable overlap. So, better denoising should exploit strong inter-color correlations present in typical color images. So here a method is proposed for denoising color images corrupted by additive white Gaussian noise, that exploits inter-channel color dependency to obtain a higher performance and also provides higher efficiency, both quantitatively and qualitatively, as well as lower computational complexity. The rest of the paper is structured as follows. Section 2 summarizes important works related to image denoising. Section 3 includes the proposed method, and the details of implementation is described in Section 4.Simulation results ans discussions of the proposed method are given in Section 5 and Section 6 concludes the paper.

\section{RELATED WORKS}

Various methods are developed to reduce Gaussian noise from digital images. This Section includes a description of some of the important works done in the area of image denoising and restoration.

S. Grace Chang et.al. [10], introduced a method in which threshold value is modified adaptive to the spatially changing statistics of the image. Each wavelet coefficient is modeled as a random variable of a generalized Gaussian distribution with an unknown parameter. For estimating parameter to each coefficient, context modeling is used to adapt the thresholding strategy. In this method adaptive thresholding gives a more accurate reconstruction and retains better sharp edges.

J. Portilla et.al.[11], have introduced a method known as the BLSGSM method, which models the neighborhoods of coefficients at adjacent positions and scales as Gaussian scale mixture and applies the Bayesian least squares estimation technique to update the wavelet coefficients. The method is one of the benchmarks in the denoising literature because of its outstanding PSNR performance.

A method that integrates bilateral filtering and wavelet thresholding has been proposed by Ming Zhang et.al [12], which helps in eliminating the coarse-grain noise in images. In this framework, an image is decomposed into low- and high-frequency components. Then bilateral filtering is applied on the approximation subbands and wavelet thresholding applied on the detail sub bands. As the selection of filter parameter is an important issue with bilateral filtering, this method also proposes an empirical method for the optimal bilateral parameter selection in image denoising.

A switching bilateral filter has been introduced by Chih-Hsing et.al. [13] that can remove both additive Gaussian noise and the impulse noise. A reference median is selected to determine whether the current pixel is noisy or not. In order to find the proper reference median, properties such as edge or texture in the current window should be understood and a sorted quadrant median vector is defined to recognize edge and texture. This method outperforms other filters, both in PSNR and visually. This method shows excellent performance in the simultaneous removal of both impulse and Gaussian noise. Hancheng Yu et.al. [14], proposed a method that combines the bilateral filter and the non-local means algorithm to improve the restoration performance for the real noisy images. This filter can group the similar neighbors efficiently based on the edge direction, the edge magnitude and the block distance non-minimal suppression. In the edge region, it preserves the image features and uses the nonlocal bilateral filter. In the non-edge region, reducing the noise level is more important, and local neighborhood-based bilateral filter is used. Performance of this filter is really good for real noisy images corrupted by Non Gaussian noise.

The method proposed by Tuan-Anh Nguyan et.al. [15] includes spatially adaptive denoising algorithm for a single image corrupted by Gaussian noise. This approach uses local statistics such as local weighted mean, local weighted activity, and local maxima to detect the noise. A spatially additive Gaussian filter is used for suppressing the additive Gaussian noise. In this proposed approach, the parameters like computational cost, over-smoothness, detection error, smoothing degree of re-constructed image are taken in to an account to effectively remove the noise components.

In the image denoising algorithm [16] proposed by C. Kervrann et.al. patch-based weights and variable window size are jointly used. This method attempts to associate with each pixel the weighted sum of data points within an adaptive neighborhood, in a way that it balances the accuracy of approximation and the stochastic error, at each spatial position. This unsupervised method doesn?t use any computational intensive training algorithm. Size of the window and control parameters are estimated from local image statistics. The algorithm is able to denoise both piecewise-smooth and textured natural images since they contain enough redundancy.

Priyam Chatterjee et.al. [17] have introduced clustering based denoising with locally learned dictionaries in which a novel patchbased framework is developed for image denoising through local geometric representations of an image. A suitable basis is then learned in each cluster which can be achieved by performing a simple principal component analysis (PCA) on the member patches of each cluster. The denoised estimate of pixel intensities is finally obtained using the steering kernels and the locally learned dictionaries. Performance of this method is comparable with current state -of -art denoising algorithms.

The method proposed by Lei Zhang et.al. [18] includes an efficient PCA-based denoising method with local pixel grouping (LPG). In this method a pixel and its nearest neighbors are modeled as a vector variable. Then the training samples of this variable are selected by grouping the pixels with similar local spatial structures to the underlying one in the local window. With this local pixel grouping procedure, the local statistics of the variables can be accurately computed so that the image edge structures can be well preserved after shrinkage in the PCA domain for noise removal. 
K. Dabov et.al. [19] have proposed a hybrid approach for image denoising based on an enhanced sparse representation in transformdomain. For achieving enhancement of the sparsity similar 2-D fragments of the image are grouped into 3-D data arrays. Then Collaborative Filtering (which is a special procedure developed to deal with these 3-D groups) is performed by Shrinkage in Transform Domain. A final estimate of the true-image can be computed by aggregating all of the obtained local estimates using a weighted average. This method ranks among the best performing methods that define the current state of the art.

An approximate digital implementations of the ridgelet transform and the curvelet transform is presented by J. L. Starck et.al [20]. The proposed method offer exact restoration, steadiness against perturbations, ease of implementation, and reduced computational complexity. In 2- D fast discrete curvelet transform (2D FDCT) with soft thresholding for image denoising, by A.A Patil et.al [21] a subband dependent threshold is implemented with curvelet transform, based on generalized Gaussian distribution modeling of subband coefficients. Denoising algorithm uses BayesShrink soft thresholding to improve smoothness and for better edge preservation.

Ruomei Yan et.al. [22] have introduced an improved NLM method. In this paper, moment invariants based k-means clustering is applied on the Gaussian blurred image, which provides better classification. Moreover this method also integrates rotationally invariant block matching (RIBM) which provides the rotation invariant weight calculation within each cluster. The simulation results show that this method outperforms the original NLM in terms of both quantitatively and visual quality. A patch-based locally optimal Weiner filter (that can be expressed as a summation of NLM and residual filtering) is proposed by Priyam Chatterjee et.al [23] to denoise grayscale images. The performance of this method is equal to or sometimes exceeding current state of the art, visually and quantitatively.

A segmentation based approach to automatically estimate the noise level from a single image is proposed by C. Liu, R et.al. [24]. A noise level function (NLF), is introduced which is a continuous function describing the noise level as a function of image brightness. An upper bound of the real NLF is estimated by finding the lower envelope of the standard deviations of image variance per segment. For denoising, the chrominance of color noise is significantly removed by projecting pixel values onto a line fit to the RGB values in each segment. By formulating and solving a Gaussian conditional random field, the noise is removed. This approach can generate sharper edges, produce smoother flat regions and preserve suitable texture details. Tolga Tasdizen [25] has introduced a method in which variation of the nonlocal means (NLM) image denoising algorithm that uses principal component analysis (PCA) is presented to achieve a higher accuracy while reducing computational load. In this approach, by using PCA, image neighborhood vectors are first projected onto a lower dimensional subspace. The dimensionality of this subspace is selected automatically using parallel analysis. Accordingly, neighborhood similarity weights for denoising are computed using distances in this subspace rather than the full space. Use of lower dimensional projections for computing similarity weights results in better accuracy in addition to reduced computational cost.

\section{PROPOSED METHOD}

Literature survey reveals that, despite the richness of works on denoising grayscale images, very little work done for color image denoising.

As with grayscale images, the prior knowledge of both uncorrupted image and contaminating noise play a critical role in recovery techniques of color images also. There are methods developed to, perform denoising by explicitly modeling the color information at each pixel. However it is found that frequencies at which the human eye perceives each of the red, green, and blue (RGB) colors have considerable overlap. Therefore in contrast to grayscale images, inter-channel dependency in color images offers a new opportunity for better resolving the intensity uncertainty from noisy samples. Consequently, many color denoising methods take into account such dependencies, either implicitly or explicitly. For exploiting the correlation between color components a luminancechrominance transformation of an RGB natural image corrupted by Gaussian noise is performed here, denoting the luminance with $\mathrm{Y}$ and the chrominances with $\mathrm{Cb}$ and $\mathrm{Cr}$. The principal advantage of this transformation is decoupling of luminance and color information and because of this decoupling, the luminance component of an image can be processed without affecting its chrominance components. Also, the luminance, Y, contains the most of the valuable information such as edges, shades, objects, textures, patterns etc. Hence, it is only needed to use information from the luminance component for clustering. So by taking into account the correlation across the color components, the denoising performance of this method can be improved substantially.

\subsection{Outline of the proposed method}

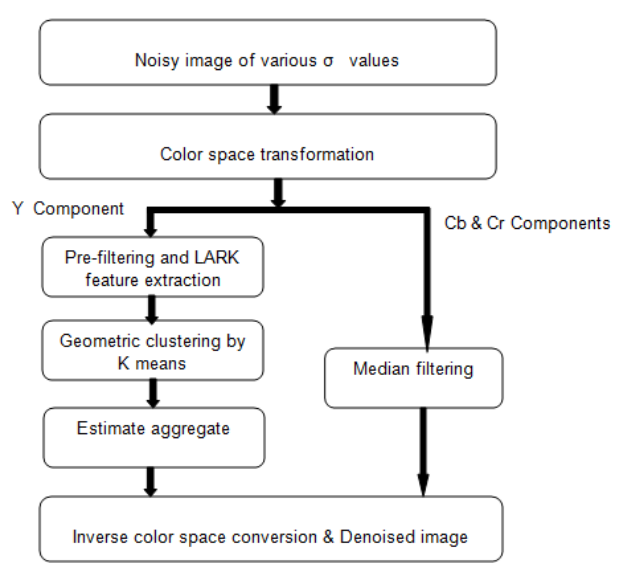

Fig. 1. Block Diagram of the Proposed method.

In this approach, at first the noisy RGB color image having various noise standard deviations $(\sigma)$ is first transformed in to $\mathrm{YCbCr}$ color space and luminanance and chrominance components are separated. The luminance $(\mathrm{Y})$ component is then pre-filtered using a Weiner filter. The pre-filtered component is then divided into different overlapping patches and for each patch, LARK (Locally Adaptive Regression Kernel) features are extracted, based on which $\mathrm{k}$-means clustering is done. As the next step the mean and the covariance of the patches within each cluster will be estimated and for each patch, photometrically similar patches will be identified. 
Then weights are computed based on their similarity to the reference patch and the average of weighted pixels is calculated. Since overlapping patches are used, multiple estimates are obtained for pixels lying in the overlapping regions. So these multiple estimates are then optimally aggregated to obtain the final denoised Y component. $\mathrm{Cb}$ and $\mathrm{Cr}$ components are applied to median filtering and these components are combined and converted back to RGB image to obtain the final denoised image.

\section{IMPLEMENTATION DETAILS}

Since this is a patch-based frame work, the noisy image has to be divided into different patches. Here the patch-size and number of clusters are fixed as $11 \times 11$ and 15 respectively.The original,noise free image is subjected to various levels of Gaussian noise.The noise standard deviation used here are 10,15 and 25 .

\subsection{Color space transformation}

Here, the RGB color image whose color information is correlated, is denoised by an appropriate color-space conversion where the information between color spaces can be largely de-correlated. ie, the RGB color image is transformed into a luminancechrominance $(\mathrm{YCbCr})$ color space. Thus inter-color correlation is exploited in this frame work. Since the luminance $\mathrm{Y}$ is an additive combination of $\mathrm{R}, \mathrm{G}$ and $\mathrm{B}$ color components it preserves the high-frequency image content. The color differences $\mathrm{Cb}$ and $\mathrm{Cr}$ are obtained by subtracting the color components and contain mostly low-frequency information. Since the luminance component contains most of the valuable information such as edges, shades, objects, texture patterns, etc. similar patches can be identified using the Y component, to which the human visual system is more sensitive. Hence, in this approach only the noisy image luminance is required to perform geometric clustering. Thus only the $\mathrm{Y}$ component of the image is required for subsequent processing by the PLOW filter.

\subsection{Pilot Estimate and Lark Feature Extraction}

For obtaining bettter denoising result the input image must be prefiltered. The Y component is prefiltered using a Weiner filter to get a pilot estimate and subsequently subjected to LARK feature extraction for performing clustering.Although $\mathrm{Cb}$ and $\mathrm{Cr}$ have only low-frequency information the are also subjected to median filtering to improve the image quality.Since the PLOW filter considers both geometric and photometric similarity across image patches, first the Y component must be clustered into geometrically similar regions. The features used for clustering should achieve the intended segmentation. Also it is important to choose a meaningful distance metric that captures similarities between the chosen features. For performing practical clustering, it is necessary to identify the features that capture the underlying geometric structure of each patch from its noisy observations and such features need to be robust to the presence of noise, as well as to differences in contrast and intensity among patches exhibiting similar structural characteristics.

In this approach, the Locally Adaptive regression Kernels (LARKs)developed by Takeda et al. [27], are used as features for geometric clustering and object detection and these regression kernels can capture the underlying local structure of the data extremely well even in the presence of significant distortions. The fundamental component of this approach is the calculation of the local steering kernel (LSK), that measures the local similarity of a pixel to its neighbors both geometrically and photometrically. Here the key idea is to robustly obtain local data structures by analyzing the photometric (pixel value) differences based on estimated gradients and use this structure information to determine the shape and size of a canonical kernel and these kernels form excellent descriptors of the underlying image geometry $[17,26]$.

As the choice of the kernel function has only a small effect on the accuracy of estimation, any kernel function can be used.So here preference is given to the differentiable kernels with low computational complexity such as the Gaussian kernel. LARK effectively and efficiently captures local geometric structure by taking advantage of self-similarity based on gradients. For measuring the similarity of two pixels, instead of Euclidean distance geodesic distance is used. These weight vectors, based on image gradient information, are used here, as feature vectors to perform clustering.

\subsection{Geometric Clustering}

The first step of the proposed denoising method is grouping together patches of similar geometric structure. Presence of noise makes identifying of such structure difficult. Moreover, such grouping needs to be independent of the patch intensities. Feature vectors opted for clustering are LARK, with that, K-means algorithm is used to cluster the noisy image into regions containing geometrically similar patches because, K-Means clustering using LARK features provides a reasonably accurate clustering, even when the input image is contaminated by considerable noise. Although many clustering methods are suitable for this particular clustering purposes, K-Means clustering is used here due to its simplicity and efficiency. K-Means requires as input, the features (normalized LARK vectors in this case) and the number of clusters. The number of clusters will vary across images based on the variance in the edge orientation and strength that an image exhibits.

The selection of the number of clusters has significant importance. If the number of clusters are too few, that may lead to clustering of structurally dissimilar patches and may results in incorrect estimation of the moments. At the same time, if too many clusters are there,that may lead to too few patches within each cluster, and thus makes the moment estimation process less robust.

\subsection{Estimate Aggregate}

4.4.1 Estimate Cluster Moments. Once the image is segmented into structurally similar regions, the moments, namely, mean and covariance, are estimated from the noisy member patches of each cluster. The mean of the underlying noise-free image can be approximated by the expectation of the noisy patches within each cluster as [23]

$$
\hat{Z}=E\left[y_{i} \in \Omega_{k}\right] \approx 1 / M_{k} \sum_{y_{i} \in \Omega_{k}} y_{i}
$$

where $\Omega_{k}$ denotes the k-th cluster with cardinality $M_{k}$. The accuracy of this estimate is dependent on $M_{k}$. If the number of patches are less, the mean vector will remain noisy. The covariance matrix $C_{z}$ is also estimated [23] from the noisy patches within the cluster. For this, of the relation between the covariance of the noisy $\left(C_{y}\right)$ and noise-free patches $\left(C_{z}\right)$ from the equation below are used.

$$
C_{y}=C_{Z}+\sigma^{2} I
$$

where $\sigma^{2}$ is the noise covariance and I is the $n \times n$ identity matrix. Thus it is necessary to first estimate the covariance of the noisy patches $\left(C_{y}\right)$. Many covariance estimators exist and the simplest of 
such estimators,i.e., the sample covariance, is the maximum likelihood estimate. After estimating the sample covariance $\hat{C}_{y}$, the covariance of the underlying noise-free patches is estimated as [23]

$$
\hat{C}_{z}=\left[\hat{C}_{y}-\sigma^{2} I\right]_{+}
$$

where $\sigma^{2}$ is the noise covariance and $[X]_{+}$denotes matrix $\mathrm{X}$ with its negative eigenvalues replaced by zero (or a very small positive value). The covariance of the underlying noise-free patches are estimated using the noise covariance and a matrix with its negative eigen values replaced by zero or a very small positive value. For this, it is required to accurately estimate the noise standard deviation first. Using the vectorized form of the gradient of the input image gradient is estimated. The gradient image is calculated using the vectorization operation (column- or row-wise) and the convolution operation simply implies the addition of the forward gradients in the horizontal and vertical directions.

4.4.2 Calculate Weights for Similar Patches. As the next step, it is needed to identify patches that are photometrically similar to a given reference patch within the noisy image. After the similar patches are identified for a given reference patch, denoising is performed with the more similar patches exerting greater influence in the denoising process. For this, the weights for all the photometrically similar patches have to be computed. Also, weight is related to the inverse of the expected squared 12 distance between the underlying noise-free patches and a noise term and it is calculated as [23]

$$
w_{i j} \approx 1 / \sigma^{2} \exp \left\{-\left\|y_{i}-y_{j}\right\|^{2} / h^{2}\right\}
$$

The smoothing parameter is a positive parameter that controls the rate at which the contributing factor is driven to zero as the patches become less similar. Though this parameter is tunable in general, it is kept fixed in this algorithm.

4.4.3 Aggregate the Multiple Pixel Estimates. The filter is run on a per-patch basis, resulting denoised estimates for each patch of the noisy input. The Y component of the noisy image is divided into different overlapping patches to avoid the block artifacts at the patch boundaries. Therefore, multiple estimates for the pixels lying in the overlapping regions is obtained. Hence it is necessary to estimate the denoised patch and calculate the aggregate of multiple estimates to reconstruct the image. Although the simplest method of aggregating such multiple estimates is to average them, such naive averaging will lead to an oversmoothened image. Therefore, in keeping with earlier formulation, the multiple estimates can be combined in an LMMSE scheme that takes into account the relative confidence in each estimate.

\subsection{Inverse Color space transformation}

Here a denoised estimate of the original image in the RGB color space has to be generated. This is achieved by the inverse color transformation of the denoised estimate of $\mathrm{Y}$ component resulted from the PLOW filter, and the prefiltered $\mathrm{Cb}$ and $\mathrm{Cr}$ components.

\section{RESULTS AND DISCUSSION}

Here to validate the proposed method, denoising algorithm is evaluated through experiments on various images at different noise levels.For testing, some of the images available at Berkley Segmentation Database have been used. The original, noise free image is given as input to the algorithm. Then the performance of the filter is analysed by adding the White Gaussian Noise with standard deviation $(\sigma) 5,10$ and 25 . Image quality under the proposed method is evaluated in terms of MSE,PSNR and SSIM values. The figure shows the fifteen images, covering different types of objects and scenes, selected from the Berkeley image Segmentation Database(BSD) to evaluate the proposed algorithm. The file names (numbers) are shown beneath each picture.

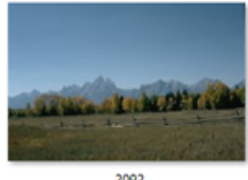

2092

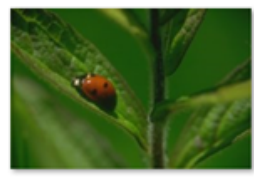

35058

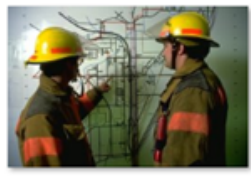

3024

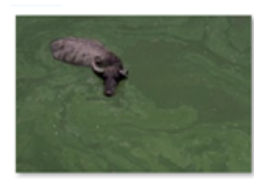

80099

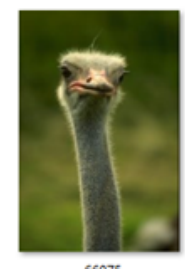

66075

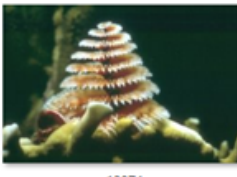

12074

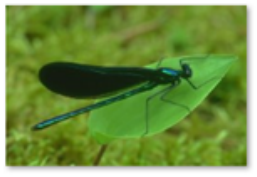

35070
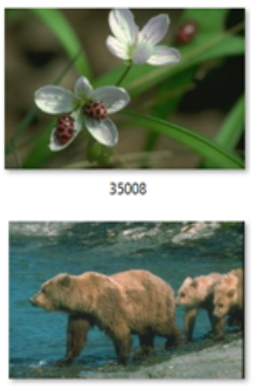

100075

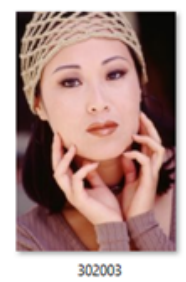

300003
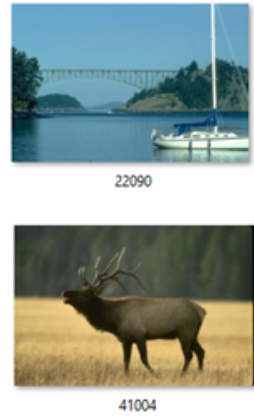

41004

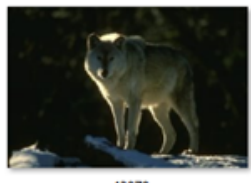

4207

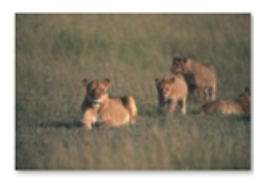

105053

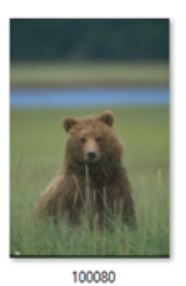

Fig. 2. Test Images from BSD

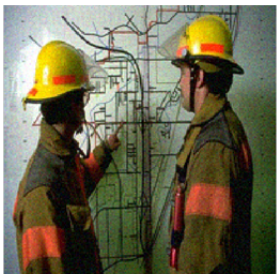

(a)

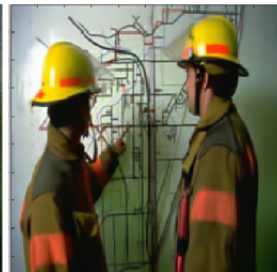

(b)

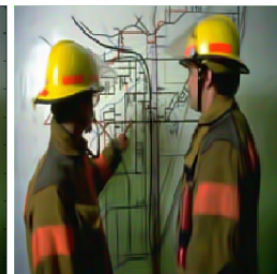

(c)
Fig. 3. A sample noisy image (a) and its denoised versions when denoising each color channel independently (b) and with proposed method (c)

The figure 3(a) shows a sample image from BSD when $\sigma=10$. When denoising each color channel independently (3(b)) a image with PSNR value of 31.7324 . With the proposed method (3(c)) the PSNR value is 34.5637 . 
Table 1. Denoising performance of the proposed method in terms of MSE PSNR and SSIM

\begin{tabular}{|c|c|c|c|c|c|c|c|c||c|}
\hline Image & \multicolumn{3}{|c|}{$\sigma=5$} & \multicolumn{3}{|c||}{$\sigma=10$} & \multicolumn{2}{|c|}{$\sigma=25$} \\
\hline & MSE & PSNR & SSIM & MSE & PSNR & SSIM & MSE & PSNR & SSIM \\
\hline 2092 & 6.5700 & 40.1149 & 0.962 & 20.8582 & 35.8372 & 0.91051 & 34.3200 & 31.945 & 0.86739 \\
\hline 12074 & 4.8776 & 39.8136 & 0.98344 & 18.8158 & 35.5578 & 0.96836 & 59.1629 & 32.0052 & 0.9340 \\
\hline 22090 & 6.0417 & 40.179 & 0.97622 & 22.3212 & 35.6309 & 0.93814 & 44.8635 & 31.7403 & 0.89981 \\
\hline 23084 & 8.0492 & 39.1362 & 0.97458 & 36.1881 & 34.5637 & 0.92221 & 104.5954 & 31.1431 & 0.83925 \\
\hline 35008 & 3.6899 & 41.1708 & 0.98642 & 13.7575 & 36.5717 & 0.96407 & 31.4704 & 32.2857 & 0.92628 \\
\hline 35058 & 4.7108 & 40.3997 & 0.97302 & 10.7272 & 36.8766 & 0.94807 & 22.7753 & 33.1336 & 0.90525 \\
\hline 35070 & 4.1283 & 40.9487 & 0.97913 & 12.1552 & 36.9235 & 0.95669 & 25.9419 & 32.3628 & 0.91344 \\
\hline 41004 & 5.3972 & 40.6858 & 0.97275 & 16.6560 & 36.3662 & 0.93666 & 33.3276 & 32.0331 & 0.89062 \\
\hline 42078 & 4.1044 & 40.9216 & 0.97911 & 12.8287 & 37.2634 & 0.96307 & 47.1441 & 34.1029 & 0.91208 \\
\hline 66075 & 4.7645 & 40.8640 & 0.97764 & 17.2435 & 36.3393 & 0.93227 & 26.8391 & 32.6654 & 0.91273 \\
\hline 80099 & 7.0616 & 39.4442 & 0.93012 & 14.0885 & 36.2985 & 0.8871 & 22.3254 & 32.0385 & 0.84463 \\
\hline 100075 & 11.1497 & 37.8882 & 0.94469 & 39.2495 & 33.5175 & 0.83341 & 62.585 & 31.0178 & 0.75349 \\
\hline 100080 & 5.1413 & 40.7977 & 0.96625 & 11.6591 & 36.6532 & 0.92988 & 19.9551 & 32.0928 & 0.89288 \\
\hline 105053 & 6.9314 & 39.7813 & 0.94707 & 17.5139 & 35.8347 & 0.89194 & 32.0267 & 31.7237 & 0.8243 \\
\hline 302003 & 6.3083 & 40.1521 & 0.97991 & 23.4321 & 35.7364 & 0.95359 & 64.973 & 31.9545 & 0.90926 \\
\hline
\end{tabular}

Images are taken from Berkley image Segmentation Database and subjected to synthetic AWGN with various $(\sigma)$ values.

Table 2. Denoising numerical results when the algorithm is applied on each color channel individually (PSNR1) and when applied to proposed method (PSNR2)

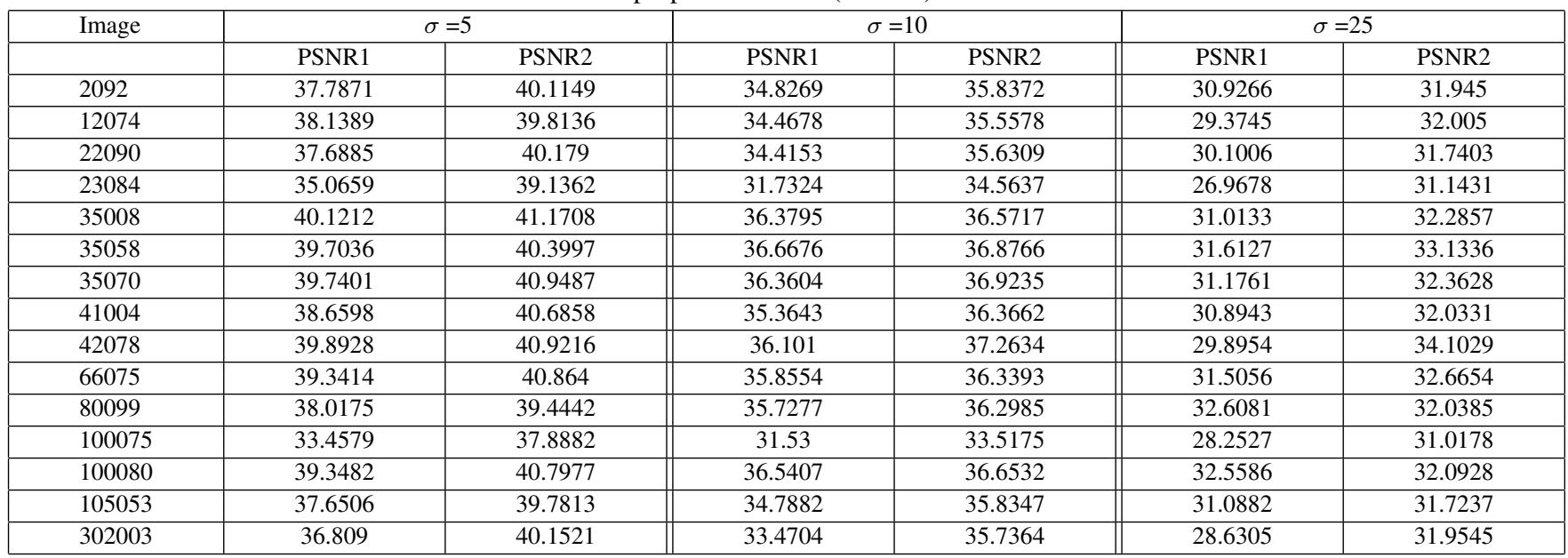

Table 1 shows the results obtained when the proposed method is tested using synthetic AWGN with constant and known noise levels; ie, with noise standard deviation $(\sigma)$ of 5,10 and 25 .

The results obtained indicate that the algorithm performs surprisingly well when $\sigma=5$ and 10. Also results are acceptable for $\sigma=25$, both visually and in terms of PSNR values. The time taken by this method to denoise an image depends on the number of clustrers $(\mathrm{K})$ used, and the noise variance. Since stronger noise typically requires more iterations, here two iterations are used for noise standard deviation greater than 5 .

In table 2, the denoising performance of the proposed method and the results obtained when the algorithm is applied on each of the color channel independently (traditional method) are shown, in terms of PSNR value. It is observed that when noise standard deviation is 5 , performance of the proposed method is extremely good for almost all the images. For $\sigma=10$ and 25 also, the proposed method yields better results compared to the traditional method. When $\sigma=$ 10 , the proposed method shows a higher performance for the image 23084. When $\sigma=25$, the performance of the proposed method with image number 100080 is not better, but comparable with the traditional method.

\section{CONCLUSION}

Image denoising is an important preprocessing task in the field of image processing and image analysis. Denoising has been a permanent research topic for engineers and scientists and one reason for it is the lack of a single technique, which can accomplish denoising for a wide class of images. This project aims to develop a method to denoise the color images corrupted by white Gaussian noise. As opposed to most color denoising algorithms, that perform denoising in each color component independently, this method performs denoising in the luminance-chrominance color space, where the information between color spaces is largely decorrelated. The similar patches are identified using the luminance component, to which the human visual system is more sensitive. Since the luminance component contains most valuble informations such as edges, textures and patterns, only the noisy image luminance is used to perform geometrical clustering. Thus much of the processing overhead is reduced. The experimental results show that the algorithm performs 
exceedingly well for small noise levels such as $\sigma=5$ and 10. Also when noise level is quite high (for $\sigma=25$ ) this method produces acceptable output, both quantitatively and visually.

This method is not very sensitive to the number of clusters when it lies within a particular range. However it may be useful to use variants of K-Means that converge to the optimal number of clusters automatically. Use of other unsupervised clustering algorithms like the mean shift method can be considered as well.

\section{REFERENCES}

[1] B Coll A Buades and J M Morel. A review of image denoising methods, with a new one. Multiscale Model. Simul, Vol.4(no.2):pp.490530, 2005.

[2] Astola J. and Kuosmanen P. Fundamentals of non-linear digital filtering. CRC Press, NewYork, Boca Raton, 1997.

[3] C. Lopez-Martnez and X. Fabregas. Modelling and reduction of sar interferometric phase noise in the wavelet domain. IEEE Trans. Geosci. Remote Sensing, Vol.40(no.12):pp.25532566, 2002.

[4] Chen Hu. Raymond H Chan. and Mila Nikolova. An iterative procedure for removing random-valued impulse noise. IEEE Signal Processing letters, Vol.11(no.12):pp.921-924, Dec 2004

[5] Siti Noraini Sulaiman. and Nor Ashidi Mat Isa. Denoisingbased clustering algorithms for segmentation of low level saltand-pepper noise-corrupted images. IEEE Trans.Consumer Electronics, Vol.56(no.4):pp.2702-2710, Nov 2010. 